\title{
Glomus tumors associated with the bone and joints: a review of 91 cases
}

\author{
Yingjie Wang", Yongbo Xiang", Yanyan Bian, Bin Feng, Yong Liu, Xi Zhou, Lizhi Zhou, Xisheng Weng \\ Department of Orthopedic Surgery, Peking Union Medical College Hospital, Peking Union Medical College \& Chinese Academy of Medical \\ Science, Beijing, China \\ Contributions: (I) Conception and design: X Weng, Y Wang; (II) Administrative support: Y Xiang, Y Bian; (III) Provision of study materials or \\ patients: L Zhou; (IV) Collection and assembly of data: Y Liu, X Zhou; (V) Data analysis and interpretation: B Feng; (VI) Manuscript writing: All \\ authors; (VII) Final approval of manuscript: All authors. \\ \#These authors contributed equally to this work. \\ Correspondence to: Xisheng Weng. No. 1 Shuaifuyuan, Dongcheng District, Beijing 100730, China. Email: xshweng@pumch.cams.cn.
}

Background: Glomus tumors (GTs) commonly affect the subungual soft tissue but rarely affect the bone and joints. Meanwhile, GTs associated with the bone and joints have been misdiagnosed for decades.

Methods: The PubMed, Embase, and Web of Science databases were searched, and primary data extracted from selected articles were quantitively analyzed.

Results: The number of male and female patients was 52 and 39, respectively, and the right side was more commonly affected than the left. The median age at onset of male and female patients was $43.61 \pm 19.20$ and $33.16 \pm 14.87$ years, respectively, and the median illness duration was $3.00(1.00,10.00)$ and $3.00(0.50$, 7.50) years, respectively. The median follow-up time was 12 months, and the median time to recurrence or metastasis was also 12 months. Of the 91 total cases, 3 had a malignant pathological type and 4 had an uncertain malignant potential. Moreover, five cases recurred postoperatively, including two malignant cases, two benign GTs, and one case of glomangiomatosis. Of the 54 patients with recorded data on trauma, 12 reported a definite history of trauma.

Conclusions: Interestingly, the history of trauma is an important cause of GTs. The most common site of extra-subungual tissue is the knee, especially in young adults. Surgical removal was the most commonly used treatment method, and radiotherapy and chemotherapy can be combined if necessary. Furthermore, patients should be followed up for at least 2 years postoperatively to monitor for possible recurrence. Fortunately, most patients with GTs have a good prognosis.

Keywords: Glomus tumor (GT); bone and joints; predilection site; treatment; prognosis

Submitted Sep 29, 2020. Accepted for publication Oct 03, 2020.

doi: $10.21037 /$ atm-20-6998

View this article at: http://dx.doi.org/10.21037/atm-20-6998

\section{Introduction}

The first record of a glomus tumor (GT) was the description and management of eight painful subcutaneous tubercles by Wood in 1812 (1). Masson is credited for the first use of the term GT, in an article published in 1924, to describe a tumor originating from a contractile neuromyoarterial body, also known as a glomus body (2). GTs are usually rare and benign tumors composed of cells similar to modified smooth muscle cells in normal hemangiospheres (3). GTs are always located in skin areas that are rich in blood vessels, such as the subungual area or deep dermis of the palms, wrists, forearms, and feet (4). Clinical diagnosis is based on the typical triad of pain, tenderness to palpation, and hypersensitivity to cold. Histopathological examination confirms the final clinical diagnosis. The main histopathological characteristic is a large number of proliferating blood vessels or 
Table 1 The statistical difference of age of onset and illness duration in knee, hand, and total for males and females

\begin{tabular}{|c|c|c|c|c|c|c|}
\hline Location & \multicolumn{2}{|c|}{ Male } & \multicolumn{2}{|c|}{ Female } & $\mathrm{P}^{\mathrm{a}}$ value & $\mathrm{P}^{\mathrm{b}}$ value \\
\hline Knee & $46.37 \pm 18.20$ & $4.00(1.00,10.00)$ & $32.70 \pm 10.61$ & $2.09(0.40,5.50)$ & 0.009 & 0.280 \\
\hline Hand & $43.73 \pm 22.46$ & $2.00(0,7.00)$ & $28.57 \pm 15.33$ & $0.33(0,20.00)$ & 0.109 & 0.963 \\
\hline Total & $43.61 \pm 19.20$ & $3.00(1.00,10.00)$ & $33.16 \pm 14.87$ & $3.00(0.50,7.50)$ & 0.004 & 0.780 \\
\hline
\end{tabular}

$\mathrm{P}^{\mathrm{a}}$, the difference of age of onset between males and females; $\mathrm{P}^{\mathrm{b}}$, the difference of illness duration between males and females.

blood sinuses, around which tumor cells showing hemangiopericytoma-like structure or solid arrangement, separated by smooth muscle bundles can be seen. Tumor cells positively express vimentin (Vimentin) and smooth muscle protein (SMA), and vascular endothelium positively express CD34 (3). GTs associated with the bone and joints are often neglected because much attention is paid to frequent and common diseases such as osteoarthritis and soft tissue injuries when patients visit orthopedic surgeons. To our knowledge, only 91 GT cases associated with the bone and joints have been reported, most likely because of the low incidence and high rate of misdiagnosis. Among the 91 cases, almost none was diagnosed as GT at the first visit, and one case was even not correctly diagnosed until 30 years after the appearance of symptoms. In most patients, clinical symptoms and physical signs are not specific. Imaging examinations such as radiography and, especially, magnetic resonance imaging (MRI) and ultrasonography can provide more evidence to help clinicians confirm the diagnosis of GTs and differentiate from neurogenic tumors, vascular tumors, pigmented nevi, epidermal cysts, lipomas, leiomyomas, sarcoidosis, Baker's cyst, and sub-patellar fat pad inflammation (5). In general, the rate of misdiagnosis and mistreatment of GTs associated with the bone and joints is very high; an important reason for this is the lack of epidemiological data, resulting in the lack of awareness of GTs among orthopedic surgeons. In this study, we reviewed the 91 reported cases, with a focus on the epidemiological characteristics of sex, age at onset, affected side, affected areas, illness duration, clinical manifestations, imaging results, and etiology in males and female patients. We also aimed to investigate the treatment and prognosis of GTs associated with the bone and joints. We present the following article in accordance with the MDAR reporting checklist (available at http://dx.doi.org/10.21037/atm-206998).

\section{Methods}

\section{Literature search and selection}

A literature search was performed using the PubMed, Embase, and Web of Science databases with the search terms ("Bones and Bone Tissue" OR "Bones and Bone" OR "Bone Tissue" OR "Bone Tissues" OR "Tissue, Bone" OR "Tissues, Bone" OR "Bony Apophyses" OR "Apophyses, Bony" OR "Bony Apophysis" OR "Apophysis, Bony" OR "Condyle" OR "Condyles" OR "Bones" OR "Bone") AND ("Glomus Tumor" OR "Glomus Tumors" OR "Tumor, Glomus” OR “Tumors, Glomus” OR "Glomangioma” OR "Glomangiomas") through November 2019. The inclusion criteria were as follows: case report(s) and case series, published in professional journals, and accessible full text.

\section{Data extraction and quality assessment}

Data extraction was independently performed and confirmed by two of the authors. The following information was extracted from each article: authors, sex, affected side, family history and trauma history, locations, size of GTs, age at onset, illness duration, pathological types, treatments, follow-up, recurrence, and metastasis.

\section{Results}

\section{Age at onset and illness duration}

The distribution of the 91 reported cases according to location was as follows: knee (41.30\%), hand (19.57\%), shoulder $(11.96 \%)$, vertebral body $(9.78 \%)$, foot $(5.43 \%)$, elbow $(4.35 \%)$, ankle $(3.26 \%)$, wrist $(2.17 \%)$, and hip $(2.17 \%)$ (Table S1). The total average age at onset was $43.61 \pm 19.20$ and $33.16 \pm 14.87$ years in male and female patients, respectively, with a significant statistical difference (Table 1, Figure 1A). The average age at onset of GTs 

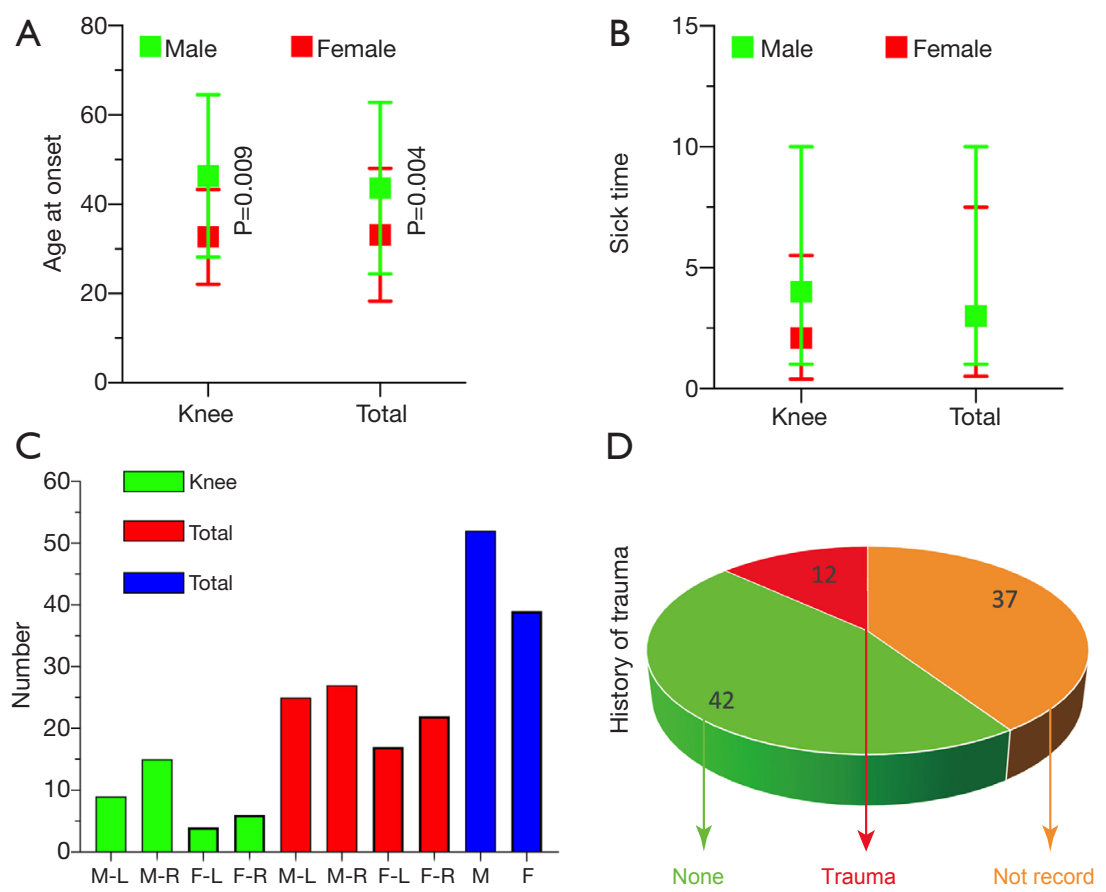

Figure 1 Epidemiological characteristics of glomus tumors (GTs) associated with bone and joints. (A) Age at onset of male and female patients with GTs in the knee and in all areas; (B) illness duration of male and female patients with GTs in the knee and in all areas; (C) number of GTs affecting the left and right sides in male and female patients; (D) history of trauma in all reported 91 cases. The figure was created by GraphPad Prism 8 software. M-L, male-left; M-R, male-right; F-L, Female-left; F-R, female-right.

associated with the knee was $46.37 \pm 18.20$ and $32.70 \pm 10.61$ years in male and female patients, respectively, with a significant statistical difference (Table 1, Figure 1A). The average sick time of male and female patients with GTs associated with the knee was $4.00(1.00,10.00)$ and 2.09 $(0.40,5.50)$ years, respectively (Table 1, Figure $1 B)$.

\section{Affected sides and etiology}

The total number of cases with the right and left sides affected was 42 and 50, respectively, and the total number of male and female patients was 53 and 39, respectively (Figure 1C). Among all patients, 12 reported a definite history of trauma, 42 denied having a history of trauma, and the remaining 37 had no recorded information on trauma history (Figure 1D).

\section{Treatments and prognosis}

GTs were completely removed in 90 patients (Table S1). One patient with GT occurring in the vertebral body of T3, involving the upper thoracic vertebral levels $\mathrm{T} 2-\mathrm{T} 4$ and extending through the neural foramen at $\mathrm{T} 3$ to the chest cavity, received radiotherapy. In this patient, GT recurred 24 months postoperatively and finally was completely surgically removed (6). In another patient, a GT in the right pedicle of T11 was successfully treated with radiofrequency ablation (7).

The distribution of pathological types was as follows: four malignant GTs, three GTs with uncertain malignant potential, with the remaining GTs being benign (Figure $2 A$ ). The postoperative follow-up rate was $67.03 \%$, and the mean follow-up time was $12[6,24]$ months. In total, five cases of GT recurred at a mean of $12[9,12]$ months postoperatively, including one malignant GT deep in the palm after resection, one benign GT in the vertebral body of T3 after radiotherapy, one malignant GT metastasis in both lungs at 3 months after surgical removal, and two multiple GTs after surgical resection (Figure 2B, Table S1).

\section{Discussion}

GTs account for $1.6 \%$ of all soft tissue tumors in the extremities (8) and are most frequently found in the skin 
A

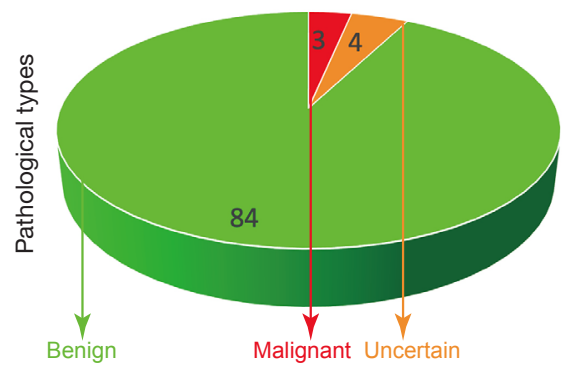

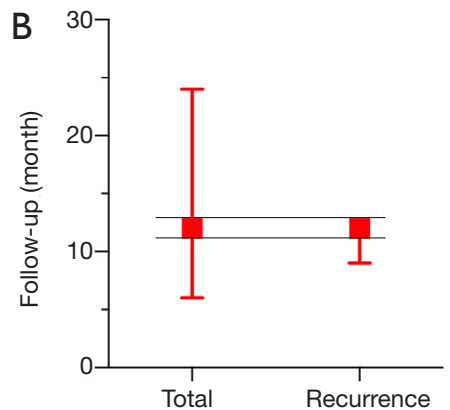

Figure 2 Pathological types of tumor and follow-up postoperatively. (A) Pathological types of glomus tumors (GTs) from 91 reported patients; (B) medians (interquartile ranges) of follow-up time from 61 patients and recurrence time from 5 patients. The figure was created by GraphPad Prism 8 software.

of the extremities. Most GTs are single tumors. The percentage tendency for multiplicity is $10 \%(9)$, and the development of multiple GTs is associated with an autosomal-dominant mutation in the glomulin gene, located on chromosome 1p21-22 (10). The incidence of multiple GTs associated with the bone and joints is $2.2 \%$ (Table S1). GTs associated with the bone and joints, including the knee, hip, ankle, shoulder, elbow, and wrist, are difficult to diagnose. Only 91 cases of GTs associated with the bone and joints have been reported thus far because of the low incidence and high rate of misdiagnosis. The lack of epidemiological data and physicians' poor understanding of the disease are the most fundamental reasons for the high rate of misdiagnosis. Moreover, epidemiological data concerning trauma history, family history, sex, affected side, affected area, age at onset, illness duration, symptoms and signs, imaging results, differential diagnosis, treatments, pathological types of GTs, and prognosis are also lacking.

Our review of the data revealed that $13.19 \%$ of GTs occurring in the bones and joints and $20 \%$ of GTs occurring in the knee are associated with a definite history of trauma. All 91 patients denied a family history of GTs. Unfortunately, trauma history was not recorded in $40.66 \%$ of all cases (Figure 1D). The common causes of the prior trauma included twisting of the joint, a fall, total knee replacement, and minor penetrating injury (Table S1). One previous study of eight cases showed a trauma history incidence of up to $50 \%$ (9), which is significantly higher than that found in our data. All data suggested that trauma, including that from total knee replacement, may be an important cause of GTs. The medical history should be recorded in detail. The diagnosis of GT should be considered in patients with a long-term history of pain that is not relieved by non-steroidal anti-inflammatory drugs.

Classic subungual GTs have been reported to occur more frequently in women, whereas ectopic locations were found to be more common in men $(11,12)$, which is consistent with our data. Furthermore, according to our data, the right side is more likely to be affected than the left side (Figure 1C). However, there was no statistical difference in the incidence rates between the right and left sides in our review. The most commonly affected areas were the knees (41.30\%), hands (19.57\%), and shoulders (12.09\%), including the cortical bone, synovium of the joint, marrow cavity, periosteum, and even nerves and vessels (Table S1).

The age at onset reflects the characteristics of the disease more closely than the age at diagnosis. A previous study reported that approximately $65 \%$ of GTs are located in subungual locations and that GTs are most commonly diagnosed between the age of 20 and 40 years (13), which is not consistent with our data. In our findings, the age at onset was older, at $43.61 \pm 19.20$ and $33.16 \pm 14.87$ years in male and female patients, respectively. Moreover, the age at onset was older in male patients than in female patients, with a significant statistical difference (Figure 1A).

Medians (interquartile ranges) were used rather than means \pm standard deviation to describe the skewed distribution of sick time. GT can be diagnosed after an average of 3 years of sick time [male patients: $3(1.0,10.0)$ years, female patients: $3(0.5,7.5$ years $)]$. In one study, the sick time ranged from 7 to 11 years (14), which is significantly longer than that reported in our review. The atypical triad signs of GTs associated with the bone and joints, low incidence of GTs, and lack of familiarity with the disease of orthopedic surgeons all contribute to the high rates of misdiagnosis and consequently delay the application 
of effective and efficient treatment for patients with GTs. Orthopedic surgeons should strengthen their understanding of the disease to enable them to make the diagnosis and treat patients as quickly as possible.

Many studies have found that extradigital GTs often present with pain but not temperature sensitivity, making these ambiguous lesions even more difficult to identify (15). For GTs in subcutaneous tissue and large GTs in deep tissue, tenderness to palpation is an obvious sign; however, this sign is not specific. The most frequent chief complaints are long-term pain and/or limited joint mobility. Accordingly, these symptoms make it easy for orthopedic surgeons to diagnose soft tissue damage in young patients and diagnose degenerative diseases, such as knee osteoarthritis and periarthritis of the shoulder, in older patients. In addition to common symptoms, some rare symptoms and signs have been reported. For example, a 59-year-old man was diagnosed with tumor-induced osteomalacia, and a zone of activity in the left ankle area was found in an octreotide scan. A further MRI revealed an in-homogenous lesion in the distal area of the left leg, posterior to the tibia. The histopathological findings after complete surgical resection led to the definite diagnosis of GT (16). Another reported case was that of a GT in the medial aspect of the right knee, which appeared on the leg of a 10-year-old boy after a fall. Unlike the localized growth of benign tumors, the tumor developed outside the skin and became mushroom-like in appearance but painless. The histopathological results finally confirmed the diagnosis of a GT, with no local recurrence after complete tumor excision (17). Clinical manifestations of GTs associated with the bone and joints are non-specific and may be very rare, requiring careful consultation and examination by orthopedic surgeons.

Optional examination methods include, but are not limited to, radiography, MRI, and ultrasonography. A radiographic examination is preferred because it is fast and economical. Although GTs' erosion of the cortical bone, corrosion of the marrow cavity, and confluence with the periosteum presented abnormal signals, radiographic results of the rest of the patients were normal.

MRI and ultrasonography are overall more efficient modalities for detection (15). Although ultrasonography is as economical, convenient, and fast as radiography, it is a routine test performed by orthopedic surgeons for diagnosing vascular diseases in the perioperative period and not for diagnosing bone and joint diseases in the clinical setting. On ultrasonography, GTs typically appear as round or ovoid hypoechoic masses and have been reported to show hypervascularity. Notably, MRI has been proven to be the most sensitive imaging modality for diagnosing GT. GTs typically show decreased signal intensity on T1weighted images and increased signal intensity on T2weighted images. When the clinical diagnosis suggests a GT, a negative MRI result should not impede excision because small tumors may be missed and lead to falsenegative results; indeed, the specificity of MRI has been estimated to be only approximately $50 \%$ (14). For patients suspected of having GT but showing negative MRI results, incision and exploration can be performed after a comprehensive evaluation. Moreover, although the characteristics of many GTs can be identified from MRI results, it is not easy to distinguish benign from malignant GTs or tumors with uncertain malignant potential (13). The results need to be confirmed using pathological staining. Imaging results should always be considered together with the size, location, border, adjacent tissue involvement, and internal characteristics of the lesion, to inform a differential diagnosis and surgical planning (5).

It was reported that only $1 \%$ of GTs are malignant (9). Moreover, there have been no major reports on the incidence of tumors with uncertain malignant potential. In our review, the incidence of GTs with uncertain malignant potential and that of malignant GTs was $4.4 \%$ and $3.3 \%$, respectively. More ample evidence is required to support the data. It is often difficult to make a definitive diagnosis based on clinical symptoms, signs, and the results of MRI and ultrasonography alone, owing to the small size of glomus tumor. In most studies, definitive diagnosis was made after hematoxylin and eosin staining coupled with immunohistochemical staining. This requires physicians to perform incisions in a timely manner after excluding other diagnoses.

Differential diagnoses for bone and joint pain and/or limited joint mobility are highly extensive. A previous study reported that only 9-20\% of extradigital GTs were correctly diagnosed by the primary physician (12). To our knowledge, only a small portion of the 91 reported cases were correctly diagnosed at the first visit (Table S1). The most common misdiagnoses were neurogenic tumors, vascular tumors, pigmented nevi, epidermal cysts, lipomas, leiomyomas, sarcoidosis, soft tissue damage, and degenerative diseases (12). Moreover, GTs associated with the knee must be differentiated from Baker's cyst, especially in the presence of knee pain in children. Furthermore, small GTs beneath the patella must also be distinguished from sub- 
patellar fat pad inflammation according to the findings of MRI and/or ultrasonography.

The reported treatments included complete surgical resection, radiotherapy, resection and adjuvant radiotherapy, resection and chemotherapy, and radiofrequency ablation. Complete surgical resection is the predominant treatment method (Table S1). The typical GT presentation is a red-purple cutaneous nodule, usually causing pain not proportional to the size of the lesion. For some GTs, such as those occurring in the spinal canal, adjuvant radiotherapy or chemotherapy could be considered after partial resection performed to retain the structure of the spinal cord and nerves. In addition, some GTs, such as those occurring in the vertebral body, require structural reconstruction to ensure the preservation of function after surgical resection.

The extent of surgical resection depends on tumor categories. GTs are classified into the following four categories: malignant GT, symplastic GT, GT of uncertain malignant potential, and glomangiomatosis. Malignant GT is characterized by a deep location and a size of $>2 \mathrm{~cm}$, or atypical mitotic figures, or moderate-to-high nuclear grade and $\geq 5$ mitotic figures/50 high-power fields. Symplastic GT is characterized by a high nuclear grade in the absence of any other malignant features. GT of uncertain malignant potential is characterized by the lack of features completely meeting the criteria of malignant GT or symplastic GT (i.e., high mitotic activity and superficial location only, or large size only). Glomangiomatosis is characterized by histological features of diffuse angiomatosis and excess of glomus cells (18). For malignant GTs, extensive resection coupled with radiotherapy or chemotherapy is preferred. Moreover, partial resection to retain important structures could be combined with adjuvant radiotherapy or chemotherapy to prevent recurrence. In addition to surgical excision, radiotherapy alone may be a potential treatment for GTs. Liu et al. reported a case of GT occurring outside the T2 paravertebral space. Treatment with radiotherapy over 3 months was chosen owing to financial considerations; however, the GT recurred 1.5 years after treatment (6). In another study, radiofrequency ablation was used to successfully cure a GT in the right pedicle of T11 (7). These reports are sparse, and further evidence is required to determine better treatments for GTs.

Patients with GTs have a good prognosis, with $94.5 \%$ experiencing no recurrence during the follow-up. The previously reported recurrence rate after surgical excision was approximately $10 \%$ (19), which is higher than that reported in our review (5.5\%). After surgical excision, pain is usually relieved immediately. Inadequate excision may result in tumor recurrence within days to weeks, and symptoms may appear only after 2-3 years (20). The postoperative follow-up rate in our review was $67.03 \%$, while the median follow-up time was 12 months. The follow-up time should be extended because our data indicated that the median time for GT recurrence was also 12 months.

More attention should be paid to patients with GTs associated with the bone and joints, particularly because these patients experience long-term pain, and suffer considerable psychological and economic burden. This study revealed that the age at onset is older in male patients than in female patients, with a significant statistical difference. Moreover, the misdiagnosis rate at the first visit can reach $100 \%$. Notably, a GT should be suspected when a patient complains of long-term pain after regular medication, especially after a trauma; however, additional cases are required to characterize this disease. Complete surgical excision is the most widely applied treatment and may be the best treatment. More evidence is needed to compare the results of surgery, radiofrequency ablation, and radiotherapy. Enhanced postoperative follow-up may better clarify a patient's prognosis and the best treatment options. Moreover, close attention should be paid to differentiating GTs with uncertain malignant potential and malignant GTs from benign GTs. Finally, more evidence is needed to identify treatment options for malignant GTs and multiple GTs.

\section{Acknowledgments}

We would like to thank Editage (www.editage.cn) for English language editing.

Funding: This study was funded by the National Natural Science Foundation of China (No. 81630064 and 81871786). The funder had no role in this study.

\section{Footnote}

Reporting Checklist: The authors have completed the MDAR reporting checklist. Available at http://dx.doi.org/10.21037/ atm-20-6998

Conflicts of Interest: All authors have completed the ICMJE uniform disclosure form (available at http://dx.doi. org/10.21037/atm-20-6998). The authors have no conflicts of interest to declare. 
Ethical Statement: The authors are accountable for all aspects of the work in ensuring that questions related to the accuracy or integrity of any part of the work are appropriately investigated and resolved.

Open Access Statement: This is an Open Access article distributed in accordance with the Creative Commons Attribution-NonCommercial-NoDerivs 4.0 International License (CC BY-NC-ND 4.0), which permits the noncommercial replication and distribution of the article with the strict proviso that no changes or edits are made and the original work is properly cited (including links to both the formal publication through the relevant DOI and the license). See: https://creativecommons.org/licenses/by-nc-nd/4.0/.

\section{References}

1. Takei TR, Nalebuff EA. Extradigital glomus tumour. J Hand Surg Br 1995;20:409-12.

2. Anley C, Vrettos B, Roche S, et al. A Glomus Tumour of the Elbow: A Case Report and Review of the Literature. Shoulder Elbow 2014;6:60-2.

3. Gombos Z, Zhang PJ. Glomus tumor. Arch Pathol Lab Med 2008;132:1448-52.

4. Stewart DR, Sloan JL, Yao L, et al. Diagnosis, management, and complications of glomus tumours of the digits in neurofibromatosis type 1.J Med Genet 2010;47:525-32.

5. Walker EA, Song AJ, Murphey MD. Magnetic resonance imaging of soft-tissue masses. Semin Roentgenol 2010;45:277-97.

6. Liu T, Zou W, Kong J, et al. Embolization in the treatment of an intraosseous glomus tumor in the upper thoracic spine complicating compression myelopathy: a case report and a literature review. Turk Neurosurg 2015;25:479-84.

7. Becce F, Richarme D, Letovanec I, et al. Percutaneous radiofrequency ablation of primary intraosseous spinal glomus tumor. Skeletal Radiol 2012;41:467-72.

8. Soule EH, Ghormley RK, Bulbulian AH. Scientific exhibits: primary tumors of the soft tissues of the extremities

Cite this article as: Wang $\mathrm{Y}$, Xiang $\mathrm{Y}$, Bian $\mathrm{Y}$, Feng B, Liu Y, Zhou X, Zhou L, Weng X. Glomus tumors associated with the bone and joints: a review of 91 cases. Ann Transl Med 2020;8(21):1460. doi: 10.21037/atm-20-6998 exclusive of epithelial tumors; an analysis of five hundred consecutive cases. AMA Arch Surg 1955;70:462-74.

9. Maxey ML, Houghton CC, Mastriani KS, et al. Large prepatellar glomangioma: A case report. Int J Surg Case Rep 2015;14:80-4.

10. Puchala M, Kruczynski J, Szukalski J, et al. Glomangioma as a rare cause of knee pain. A report of two cases. J Bone Joint Surg Am 2008;90:2505-8.

11. Clark ML, O'Hara C, Dobson PJ, et al. Glomus tumor and knee pain: a report of four cases. Knee 2009;16:231-4.

12. Lee DW, Yang JH, Chang S, et al. Clinical and pathological characteristics of extradigital and digital glomus tumours: a retrospective comparative study. J Eur Acad Dermatol Venereol 2011;25:1392-7.

13. Walker EA, Salesky JS, Fenton ME, et al. Magnetic resonance imaging of malignant soft tissue neoplasms in the adult. Radiol Clin North Am 2011;49:1219-34, vi.

14. Schiefer TK, Parker WL, Anakwenze OA, et al. Extradigital glomus tumors: a 20-year experience. Mayo Clin Proc 2006;81:1337-44.

15. Villescas VV, Wasserman PL, Cunningham JC, et al. Brace yourself: an unusual case of knee pain, an extradigital glomangioma of the knee. Radiol Case Rep 2017;12:357-60.

16. Dehghani M, Dabbaghmanesh MH, Khalafi-Nezhad A, et al. Glomus tumor as a cause of oncogenic osteomalacia. Clin Cases Miner Bone Metab 2017;14:359-62.

17. Frumuseanu B, Balanescu R, Ulici A, et al. A new case of lower extremity glomus tumor up-to date review and case report. J Med Life 2012;5:211-4.

18. Terada T, Fujimoto J, Shirakashi Y, et al. Malignant glomus tumor of the palm: a case report. J Cutan Pathol 2011;38:381-4.

19. Akgun RC, Guler UO, Onay U. A glomus tumor anterior to the patellar tendon: a case report. Acta Orthop Traumatol Turc 2010;44:250-3.

20. So SY, Kim BM, Lee SY, et al. Glomus tumor causing anterior thigh pain: a case report. Korean J Pain 2014;27:174-7.

(English Language Editor: J. Gray) 